\title{
INFLUENCIA DEL MODELO ELÁSTICO EN LA DINÁMICA DE ESTRUCTURAS ARTICULADAS
}

\author{
(INFLUENCE OF THE ELASTIC MODEL IN THE DYNAMICS OF ARTICULATED STRUCTURES)
}

\author{
Manuel Muñoz Vidal, Arquitecto y Enrique López Hernández, Dr. en Ciencias Físicas \\ Dpto. Tecnología de la Construcción. E.T.S. de Arquitectura. Universidad de La Coruña
}

ESPAÑA

Fecha de recepción: 28-IV'97

\section{RESUMEN}

Se estudia la influencia en la dinámica de una estructura articulada de barras de madera, de la aproximación a las propiedades tensión - deformación del material. Se ha adoptado un método de cálculo por diferencias finitas en el dominio del tiempo, con un modelo de masas concentradas en los nodos.

\section{SUMMARY}

Influence in the dynamics of an articulated structure of sweep wooden, of the approximation to the material tensiondeformation properties. A time-domain calculation method by finite differences has been used with a model of lumped masses in the nodes.

\section{Introducción}

Cuando en un cálculo dinámico se termina asumiendo el comportamiento no lineal del material, dejan de tener validez aproximaciones y simplificaciones ampliamente utilizados como son los modos propios de vibración, que se basan en el análisis del movimiento en el dominio de las frecuencias.

En un estudio anterior [1] ya hemos analizado en qué casos no son despreciables los efectos no lineales y, por tanto, no son admisibles hipótesis simplificativas que suponen constante el módulo de Young. En esta ocasión pretendemos estudiar, una vez que asumimos la necesidad de efectuar un análisis no lineal, en qué medida puede llegar a influir el grado de aproximación a las propiedades reales del material.

Para este estudio usaremos la estructura que describimos en el punto siguiente. Destacamos aquí que tomamos como material la madera, por poseer una gráfica de tensióndeformación eminentemente no lineal.

\section{Definición del problema}

La estructura objeto de estudio está formada por barras de madera, unidas en sus extremos mediante articulaciones y sujeta a unas condiciones de contorno o coacciones y a unas fuerzas exteriores dadas, que se resumen en la Fig. 1. Todas las barras son de idéntica longitud, de valor: 1 metro.

Sólo se consideran fuerzas aplicadas en los nudos, siendo los vectores indicados fuerzas de tipo constante. La fuerza representada con la doble flecha en el nudo 9 representa una carga variable en el tiempo, de tipo senoidal, con frecuencia angular $200 \cdot \pi$ y con dirección vertical. La amplitud $\mathbf{A}$ de esta carga variará en los distintos casos estudiados, con el fin de conocer la influencia de dicha amplitud en el comportamiento dinámico. Los segmentos de los nudos 3, 4 y 8 representan coacciones e indican en qué dirección se halla impedido el desplazamiento del nudo.

Si describimos el comportamiento de las barras en función de sus extremos, se verifica que sus ecuaciones de 


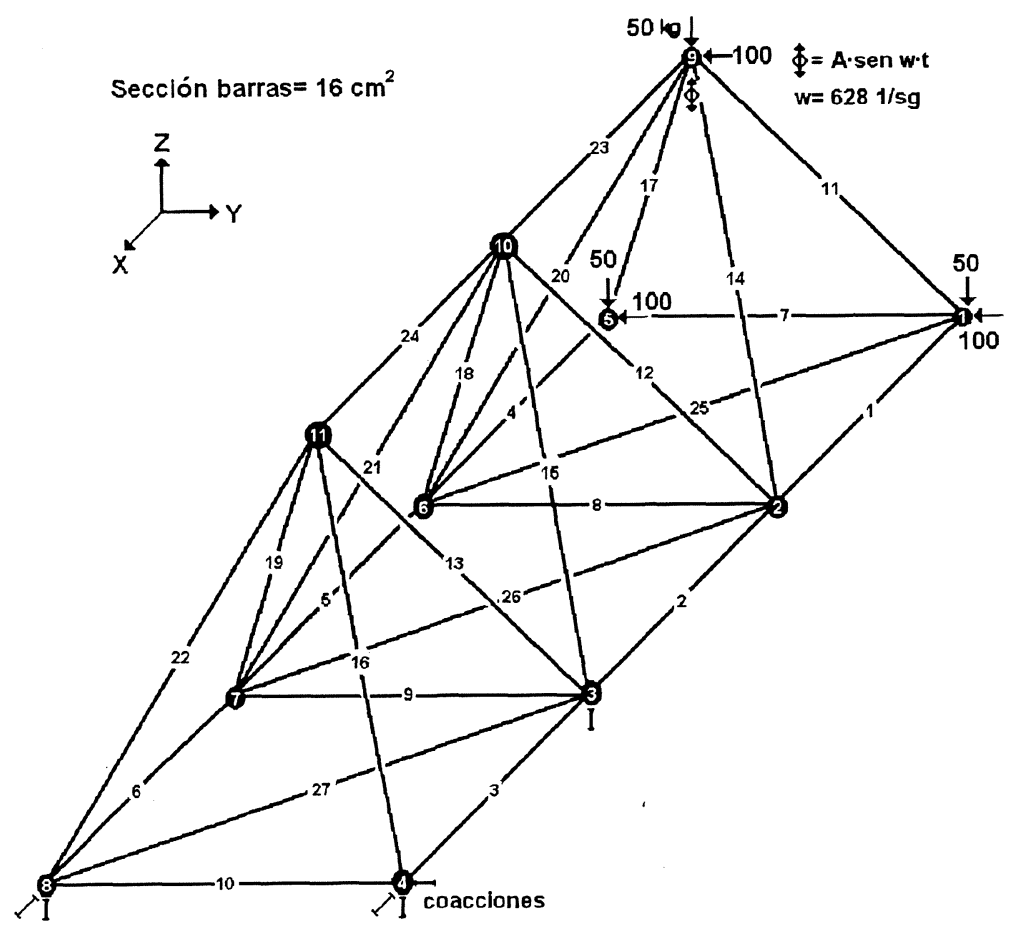

Fig. 1

movimiento resultan [2]

$$
\mathrm{D}+\mathrm{C}+\mathrm{M} \cdot \ddot{\mathrm{U}}=\mathrm{R}(\mathrm{t})
$$

siendo:

D - matriz de fuerzas internas

$\mathrm{U}$ - matriz de desplazamientos

$\mathrm{C}$ - matriz de amortiguamiento

$\mathrm{M}$ - matriz de masas consistente

$\ddot{U}$ - matriz de aceleraciones

$\mathrm{R}$ - matriz de fuerzas exteriores

Que en coordenadas locales resulta [4]:

$$
M=\frac{\rho \cdot L \cdot A}{6} \cdot\left(\begin{array}{llll}
2 & 0 & 1 & 0 \\
0 & 2 & 0 & 1 \\
1 & 0 & 2 & 0 \\
0 & 1 & 0 & 2
\end{array}\right) \quad \ddot{U}=\left(\begin{array}{c}
\ddot{u}_{1} \\
\ddot{u}_{2} \\
\ddot{u}_{3} \\
\ddot{u}_{4}
\end{array}\right)
$$

con:

$$
\begin{aligned}
& \rho \text { - densidad } \\
& L \text { - longitud } \\
& \text { A - sección transversal }
\end{aligned}
$$

Con la aproximación de masas concentradas y suponiendo los efectos de inercia asociados con los grados de libertad rotacionales despreciables, la matriz $\mathrm{M}$ la simplificamos en [3]:

$$
M=\frac{\mathrm{p} \cdot \mathrm{L} \cdot \mathrm{A}}{2} \cdot\left(\begin{array}{cccc}
1 & 0 & 0 & 0 \\
0 & 1 & 0 & 0 \\
0 & 0 & 1 & 0 \\
0 & 0 & 0 & 1
\end{array}\right)
$$

En este artículo analizamos una estructura de madera en la que los efectos de amortiguamiento y de histéresis en el material son despreciables, de tal manera que la ecuación (1) se puede reducir a:

$$
\mathrm{K} \cdot \mathrm{U}+\mathrm{M} \cdot \ddot{\mathrm{U}}=\mathrm{R}(\mathrm{t})
$$

donde:

$$
K=\frac{\mathrm{f}(\mathrm{E}) \cdot \mathrm{A}}{\mathrm{L}} \cdot\left(\begin{array}{cccc}
1 & 0 & -1 & 0 \\
0 & 0 & 0 & 0 \\
-1 & 0 & 1 & 0 \\
0 & 0 & 0 & 0
\end{array}\right)
$$

Así definido el problema, se observa que la no linealidad proviene de la matriz de rigidez $K$, debido al término $f(E)$, que representa la curvade tensión-deformación del material. En nuestro caso, el material adopta la curva que se refleja en la Fig. 2, que se ha elegido por su alto grado de alinealidad [5]

Es costumbre simplificar dicha curva reduciéndola a dos rectas, según la gráfica de la Fig. 3 (donde se consideró $\mathrm{E}^{+}=220 \cdot 10^{3} \mathrm{~kg} / \mathrm{cm}^{2}$ y $\left.\mathrm{E}^{-}=60 \cdot 10^{3} \mathrm{~kg} / \mathrm{cm}^{2}\right)$. De esta forma 


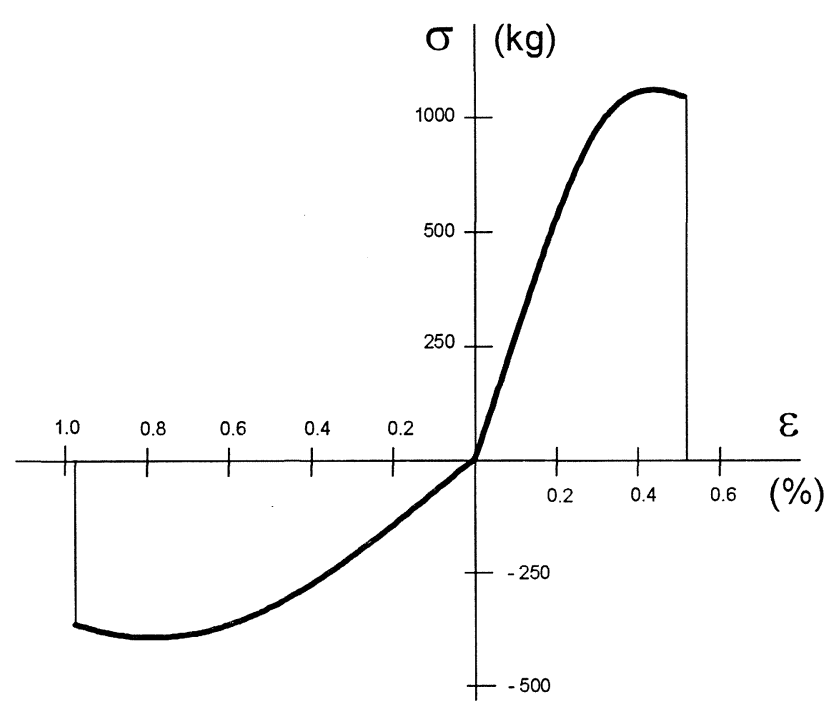

Fig. 2

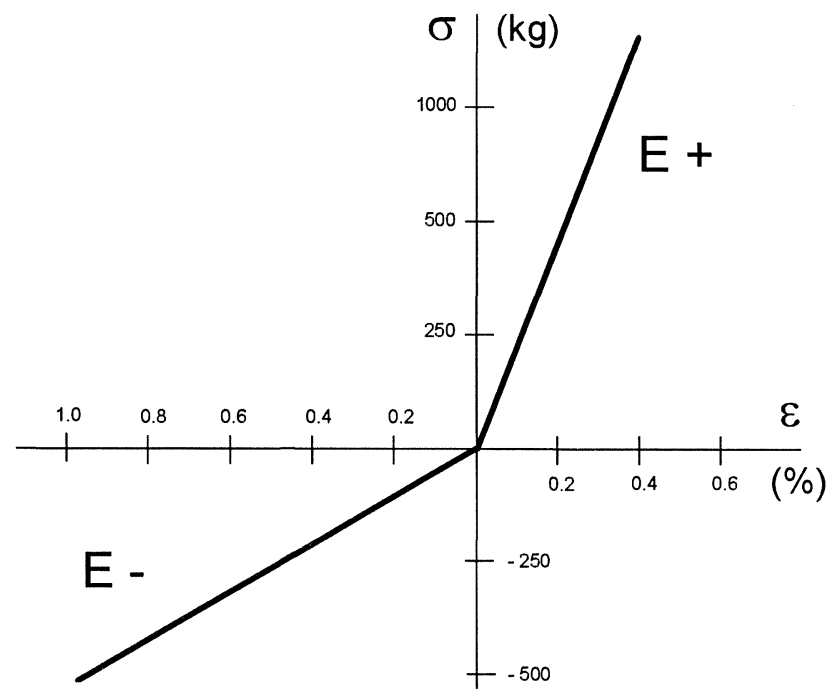

Fig. 3

el problema se reduce, ya que el módulo de Young pasa a tomar sólo dos valores constantes, uno para deformaciones positivas y otro para negativas, con lo cual, sabiendo en cada instante si la barra está traccionada o comprimida, se le asigna una matriz de rigidez $u$ otra.

Se observa que, aun siendo las dos gráficas anteriores relativamente semejantes, el esfuerzo de cálculo puede variar sustancialmente de tomar una aproximación a otra. En este artículo intentaremos estudiar en qué grado varía el comportamiento de la estructura según qué gráfica tomemos.

En ambos casos se analiza el movimiento de la estructura, debido a la fuerza dinámica, a partir del equilibrio y deformación de la estructura producido por las fuerzas estáticas. Lógicamente, la posición de equilibrio estático variará ligeramente según qué gráfica consideremos, partiendo de la base de que la estructura se halla en equilibrio estático y que el movimiento de la misma se debe, únicamente, a la carga variable

\section{Método de cálculo}

Resolvemos la ecuación (4) aprovechando que $M$ es diagonal con determinante distinto de cero, pudiéndose despejar la matriz de aceleración:

$$
\ddot{U}=M^{-1} \cdot[R-K \cdot U]
$$

Como método de resolución resolvemos la ecuación (6) mediante el método iterativo Adams-Moulton de cuarto orden. Se ha usado el método de Runge-Kutta de cuarto orden como método iniciador hasta la cuarta iteración y, a partir de ésta, se pasa al método predictor corrector de Adams-Moulton, que, con respecto al primero, presenta la ventaja de que el cálculo resulta ser más rápido. El esquema de estos métodos es:

Runge-Kutta de cuarto orden [6]:

$$
\begin{aligned}
& \mathrm{U}_{\mathrm{i}+1}=\mathrm{U}_{\mathrm{i}}+\Delta \mathrm{t} \cdot \dot{\mathrm{U}}_{\mathrm{i}}+\frac{\Delta \mathrm{t}}{6} \cdot\left(\mathrm{k}_{1}+\mathrm{k}_{2}+\mathrm{k}_{3}\right) \\
& \dot{\mathrm{U}}_{\mathrm{i}+1}=\dot{\mathrm{U}}_{\mathrm{i}}+\frac{1}{6} \cdot\left(\mathrm{k}_{1}+2 \cdot \mathrm{k}_{2}+\mathrm{k}_{3}+\mathrm{k}_{4}\right)
\end{aligned}
$$

donde:

$$
\begin{aligned}
& \mathrm{k}_{0}=\ddot{\mathrm{U}}(\mathrm{t}, \mathrm{U}) \\
& \mathrm{k}_{1}=\ddot{\mathrm{U}}\left(\mathrm{t}+\theta_{1} \cdot \Delta \mathrm{t}, \mathrm{U}+\left(\omega_{10} \cdot \mathrm{k}_{0}\right) \cdot \Delta \mathrm{t}\right) \\
& \mathrm{k}_{2}=\ddot{\mathrm{U}}\left(\mathrm{t}+\theta_{2} \cdot \Delta \mathrm{t}, \mathrm{U}+\left(\omega_{20} \cdot \mathrm{k}_{0}+\omega_{21} \cdot \mathrm{k}_{1}\right) \cdot \Delta \mathrm{t}\right) \\
& \mathrm{k}_{3}=\ddot{\mathrm{U}}\left(\mathrm{t}+\theta_{3} \cdot \Delta \mathrm{t}, \mathrm{U}+\left(\omega_{30} \cdot \mathrm{k}_{0}+\omega_{31} \cdot \mathrm{k}_{1}+\omega_{32} \cdot \mathrm{k}_{2}\right) \cdot \Delta \mathrm{t}\right)
\end{aligned}
$$

y donde:

$$
\begin{aligned}
& \omega_{0}=1 / 6 ; \omega_{1}=1 / 3 ; \omega_{2}=1 / 3 ; \omega_{3}=1 / 6 \\
& \theta_{1}=1 / 2 ; \theta_{2}=1 / 2 ; \theta_{3}=1 \\
& \omega_{10}=1 / 2 ; \omega_{20}=0 ; \omega_{21}=1 / 2 ; \omega_{30}=0 ; \omega_{31}=0 ; \\
& \omega_{32}=1
\end{aligned}
$$

Adams-Moulton de cuarto orden [6]:

Predicción:

$$
\begin{aligned}
& \mathrm{U}^{\prime}{ }_{\mathrm{i}+1}=\mathrm{U}_{\mathrm{i}}+\frac{\Delta t}{24} \cdot\left(55 \cdot \dot{\mathrm{U}}_{\mathrm{i}}-59 \cdot \dot{\mathrm{U}}_{\mathrm{i}-1}+37 \cdot \dot{\mathrm{U}}_{\mathrm{i}-2}-9 \cdot \dot{\mathrm{U}}_{\mathrm{i}-3}\right) \\
& \dot{\mathrm{U}}^{\prime}{ }_{\mathrm{i}+1}=\dot{\mathrm{U}}_{\mathrm{i}}+\frac{\Delta \mathrm{t}}{24} \cdot\left(55 \cdot \ddot{\mathrm{U}}_{\mathrm{i}}-59 \cdot \ddot{\mathrm{U}}_{\mathrm{i}-1}+37 \cdot \ddot{\mathrm{U}}_{\mathrm{i}-2}-9 \cdot \ddot{\mathrm{U}}_{\mathrm{i}-3}\right)
\end{aligned}
$$


Corrección:

$$
\begin{aligned}
& \dot{\mathrm{U}}_{\mathrm{i}+1}=\dot{\mathrm{U}}_{\mathrm{i}}+\frac{\Delta \mathrm{t}}{24} \cdot\left(9 \cdot \ddot{\mathrm{U}}^{\prime}{ }_{\mathrm{i}+1}+19 \cdot \ddot{\mathrm{U}}_{\mathrm{i}}-5 \cdot \ddot{\mathrm{U}}_{\mathrm{i}-1}+\ddot{\mathrm{U}}_{\mathrm{i}-2}\right) \\
& \mathrm{U}_{\mathrm{i}+1}=\mathrm{U}_{\mathrm{i}}+\frac{\Delta \mathrm{t}}{24} \cdot\left(9 \cdot \dot{\mathrm{U}}_{\mathrm{i}+1}+19 \cdot \dot{\mathrm{U}}_{\mathrm{i}}-5 \cdot \dot{\mathrm{U}}_{\mathrm{i}-1}+\dot{\mathrm{U}}_{\mathrm{i}-2}\right)
\end{aligned}
$$

donde los términos prima son valores intermedios relacionados por la ecuación (6)

En este tipo de esquemas de integración el error se estima del orden del intervalo de tiempo elevado a la cuarta potencia. En nuestro caso hemos tomado $\Delta t=1 \cdot 10^{-5} \mathrm{seg}$. Las ecuaciones las resolvemos para unas 65.536 iteraciones sucesivas, de tal modo que se analizaron $0,65 \mathrm{seg}$. de movimiento, con una precisión en las frecuencias de $9,58 \mathrm{seg}^{-1}$.

\section{Resultados}

Para estudiar las características del movimiento utilizamos la transformada rápida de Fourier de las aceleraciones obtenidas y las correspondientes distribuciones de densidad
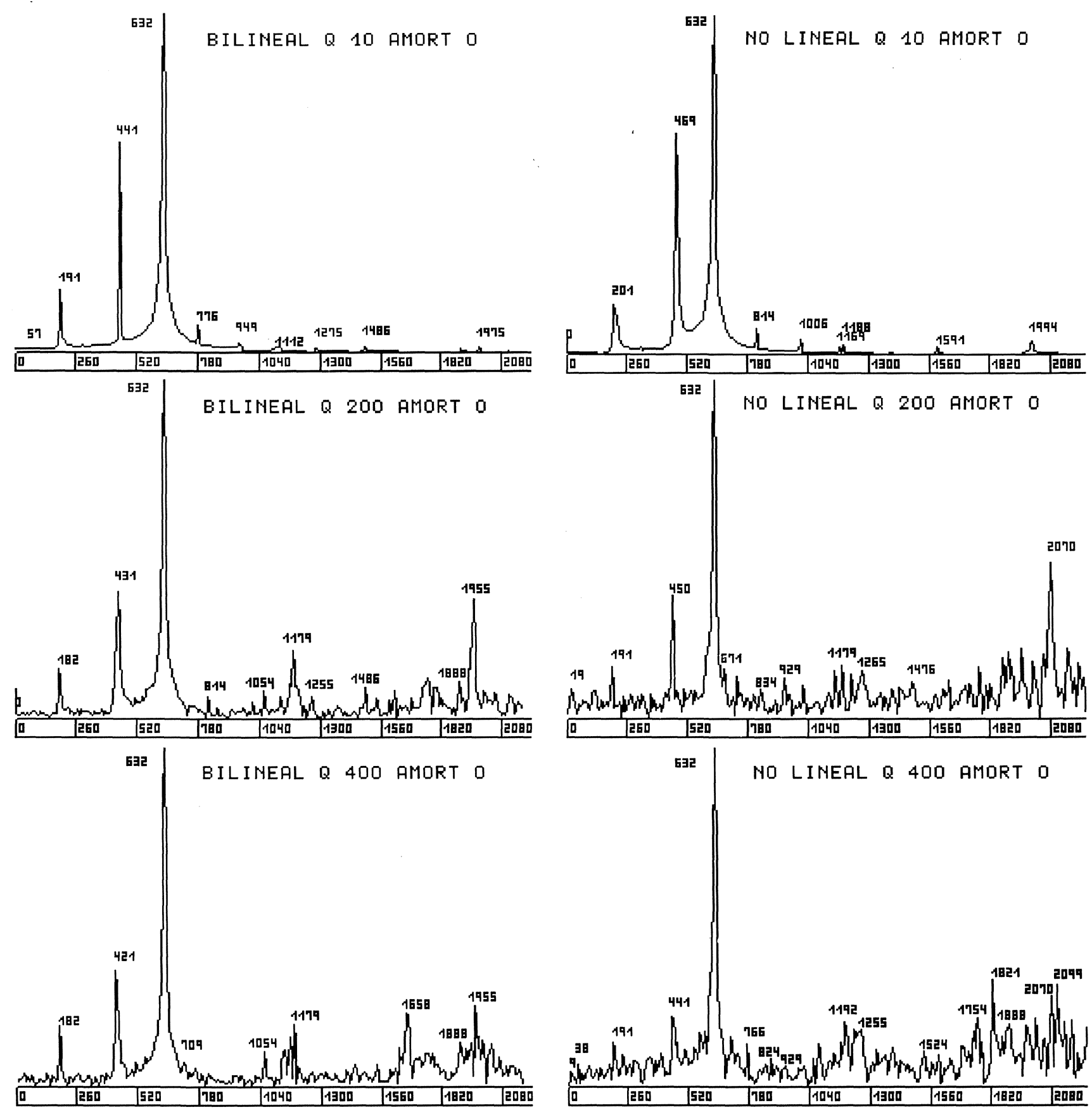

Fig. 4 
de potencia, así como las tensiones máximas que se alcanzan en cada barra en el intervalo de tiempo analizado

Se han realizado dos series de estudios: uno, con la gráfica del material bilineal y el otro, usando una no lineal poligonal que se aproxima, casi perfectamente, a la gráfica real. En los distintos casos de cada serie la única variación que se ha introducido es que la carga senoidal varía su amplitud. Para simplificar la exposición, únicamente aportaremos los resultados de los tres casos más significativos:

1.- Amplitud de la carga senoidal de $10 \mathrm{~kg}$, que representa del orden de la décima parte de las cargas estáticas.
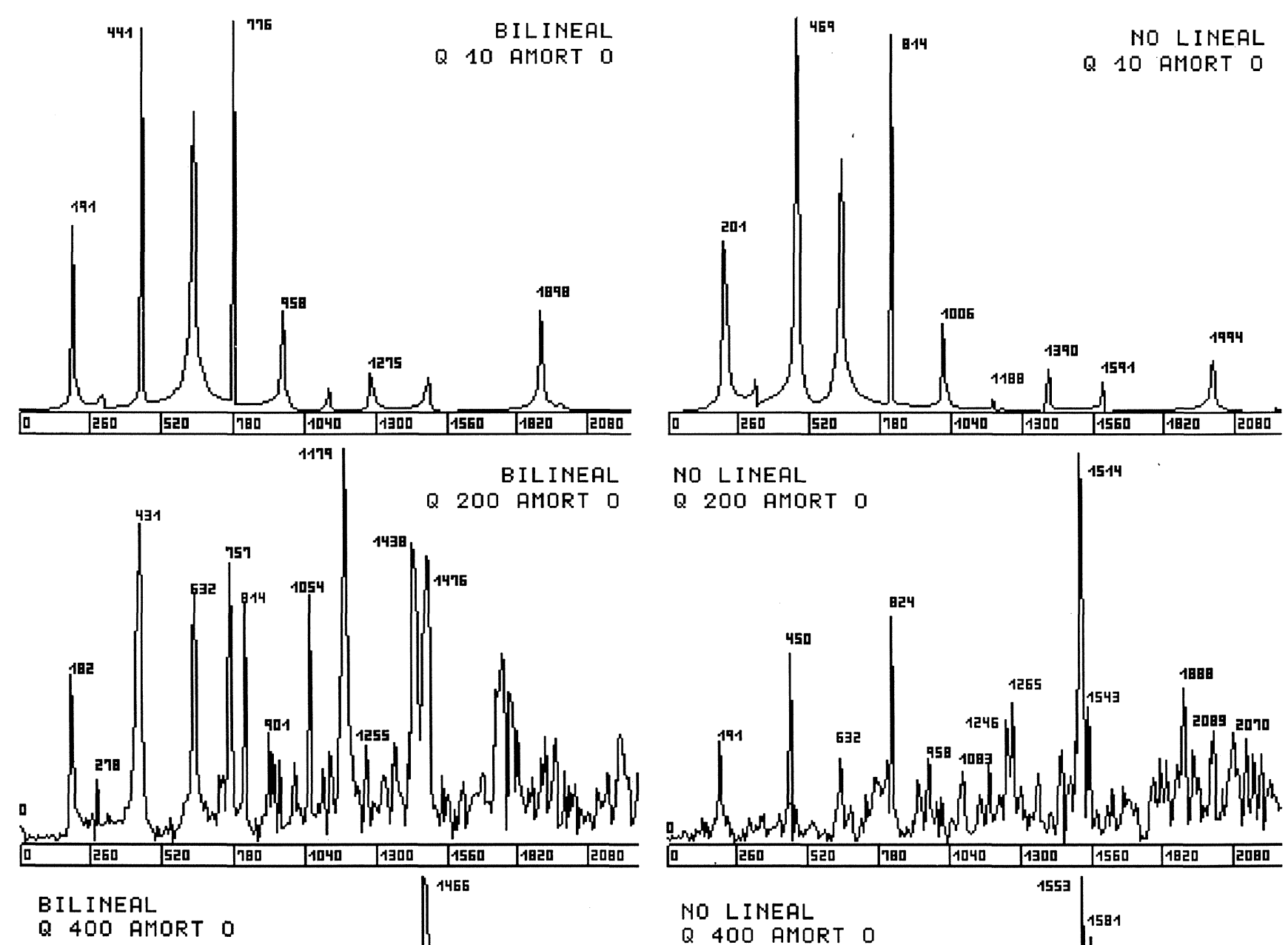

NO LINEAL

Q 200 AMORT D

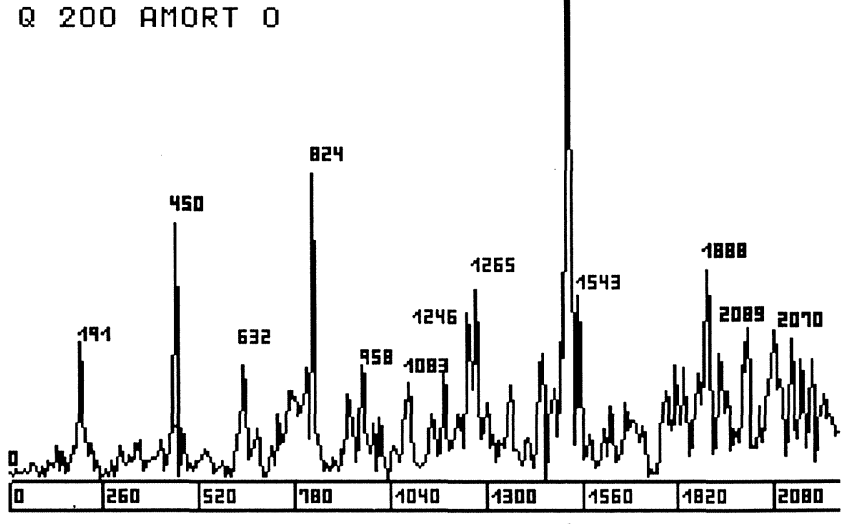

NO LINEAL

Q 400 AMORT

En la Fig. 4 se presentan los espectros del nudo 1 (ver Fig. 1) en la dirección vertical $Z$, para las dos series de materiales y para los 3 casos de carga comentados, y en la Fig. 5 para la dirección $\mathrm{Y}$ en el nudo 10, también para las dos series.

Por otra parte, resulta interesante comparar los esfuerzos axiles máximos que se producen en uno y otro caso

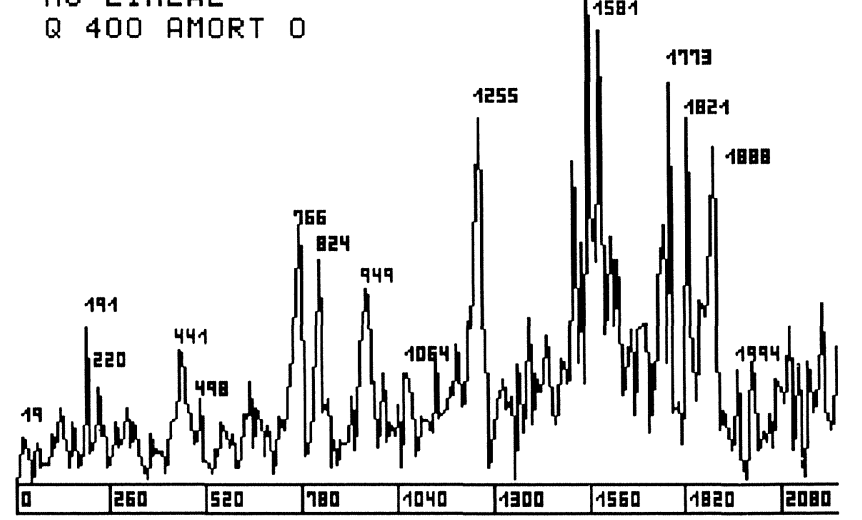

Fig. 5

2.- Amplitud senoidal de $200 \mathrm{~kg}$, de modo que la carga 3.- Amplitud senoidal de $400 \mathrm{~kg}$, teniendo, por tanto, la carga dinámica una magnitud del orden del doble de las estáticas.

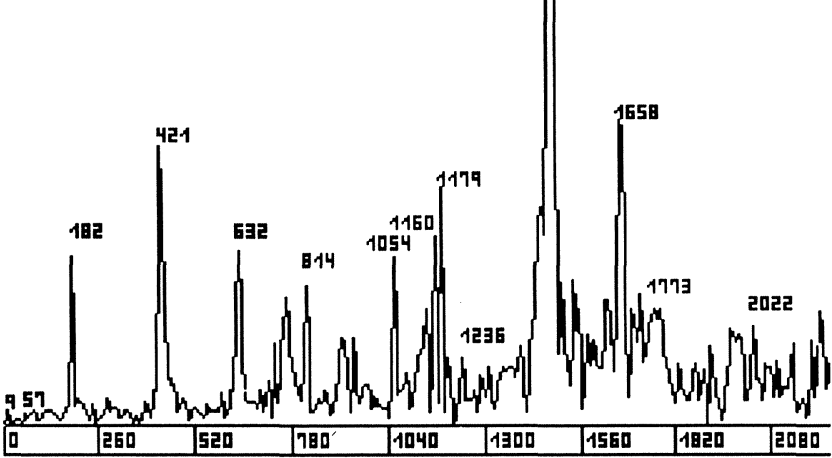


En la Fig. 6 se ofrece la comparativa de los esfuerzos axiles (en $\mathrm{kg}$ ) correspondientes a la posición de equilibrio, debidos a las cargas estáticas, para ambos tipos de diagramas.

Se aprecia, que en este estado inicial, los valores de los esfuerzos son prácticamente idéntico; ello se debe a que las tensiones de trabajo son bastante bajas (menores de 40 $\mathrm{kg} / \mathrm{cm}^{2}$ ), por lo que nos movemos en la parte de las gráficas próximas al origen de coordenadas, reflejando que la aproximación de la gráfica bilineal es bastante exacta en estos primeros tramos.

En la Fig. 7 se ofrecen los valores mínimos y máximos entre los que ha oscilado el esfuerzo axil de cada barra durante el período de estudio, debidos, por una parte, a la carga senoidal de mínima amplitud estudiada (10 kg) y, a continuación, los que originó la máxima carga dinámica (amplitud $400 \mathrm{~kg}$ ).

\section{Comentarios y conclusiones}

Se ha estudiado el comportamiento de una estructura formada por un material no lineal, sin histéresis y sin amortiguamiento, bajo los supuestos del diagrama real y uno simplificado, de tipo bilineal, para intentar estimar el grado de error que introduce esta aproximación.

Respecto al comportamiento estático se puede resumir que si la estructura se encuentra relativamente poco solicitada, las diferencias apenas serán perceptibles. Incluso si las cargas dinámicas son pequeñas, los esfuerzos máximos y mínimos que soporta cada barra apenas difieren, pudiéndose decir lo mismo del análisis en el dominio de las frecuencias, donde únicamente se puede apreciar que con la gráfica real del material las frecuencias principales no resultan tan nítidamente afinadas como en el caso bilineal.

Cuando las cargas dinámicas son del orden de las estáticas, aunque no nos alejamos mucho del origen 'de coordenadas -tensiones del orden de $80 \mathrm{~kg} / \mathrm{cm}^{2}-$, los análisis de la transformada de Fourier (Fig. 4) empiezan a diferir y cuando empleamos las mayores cargas dinámicas (amplitud $=400 \mathrm{~kg}$, tensiones del orden de $120 \mathrm{~kg} / \mathrm{cm}^{2}$ ), podemos concluir que se observa una clara diferenciación de los puntos que corresponden a las frecuencias principales del modelo no lineal con respecto el modelo bilineal. Cuando analizamos los esfuerzos que se nos producen en este último caso (Fig. 6), vemos que incluso las diferencias se acrecientan mucho más, siendo abundantes las barras en las que los axiles difieren en proporciones del orden del doble y del triple.

Podemos concluir, por tanto, que, con cargas dinámicas importantes, es preciso considerar el diagrama real del material cuando éste es altamente no lineal, tanto en lo que atañe al comportamiento de la estructura en el dominio de las frecuencias como por la estimación de los esfuerzos, donde se pueden producir fácilmente errores del orden del triple de los valores máximos.

\begin{tabular}{|l|r|r|}
\hline Barra & Bilineal & No lineal \\
1 & 176.48 & 176.45 \\
2 & 402.85 & 402.79 \\
3 & 624.26 & 624.27 \\
4 & -24.02 & -24.04 \\
5 & -398.99 & -399.02 \\
6 & -774.03 & -774.05 \\
7 & 75.51 & 75.50 \\
8 & 200.13 & 200.13 \\
9 & 196.34 & 196.41 \\
10 & 74.63 & 74.67 \\
11 & 60.46 & 60.48 \\
12 & -31.96 & -31.94 \\
13 & -547.32 & -547.41 \\
14 & 32.14 & 32.11 \\
15 & 31.21 & 31.18 \\
16 & 549.51 & 549.55 \\
17 & 60.65 & 60.66 \\
18 & 213.60 & 213.62 \\
19 & 214.03 & 214.03 \\
20 & -213.67 & -213.68 \\
21 & -213.24 & -213.26 \\
22 & -217.27 & -217.21 \\
23 & 124.33 & 124.34 \\
24 & 272.61 & 272.66 \\
25 & -283.22 & -283.22 \\
26 & -283.21 & -283.21 \\
27 & 18.88 & 18.87 \\
& \multicolumn{3}{|c|}{} \\
\hline
\end{tabular}

Fig. 6

\section{BIBLIOGRAFÍA}

[1] LÓPEZ Y M. MUÑOZ: "Influencia de la no linealidad del material en la dinámica de estructuras articuladas de madera". Informes de la Construcción. $n^{\circ} 444$ julio/agosto 1996

[2] S. S. RAO: "Mechanical Vibrations" $3^{\mathrm{a}}$ Ed. AddisonWesley Co. Reading, Massachusetts. 1995. Chap. 1-2

[3] M. L. JAMES, G. M. SMITH, J. C. WOLFORD, P. W. WHALEY: "Vibration of Mechanical and Structural Systems". Harper\&Row Publishers, New York 1989. Chap. 6-7

[4] RAMÓN ARGÜELleS ÁlVAREZ: "Cálculo de Estructuras”. E.T.S.I. de Montes, Madrid 1981. Cap. XXI

[5] RAMÓN ARGÜELLES ÁlVAREZ Y FRANCISCO ARRIAGA MARTITEGUI: "Diseño y cálculo de estructuras de madera", C.O.A.M.

[6] Curso de "Métodos avanzados de cálculo dinámico de estructuras". E.T.S. de Ingenieros de Caminos, Canales y Puertos. Universidad de La Coruña. junio 1996 


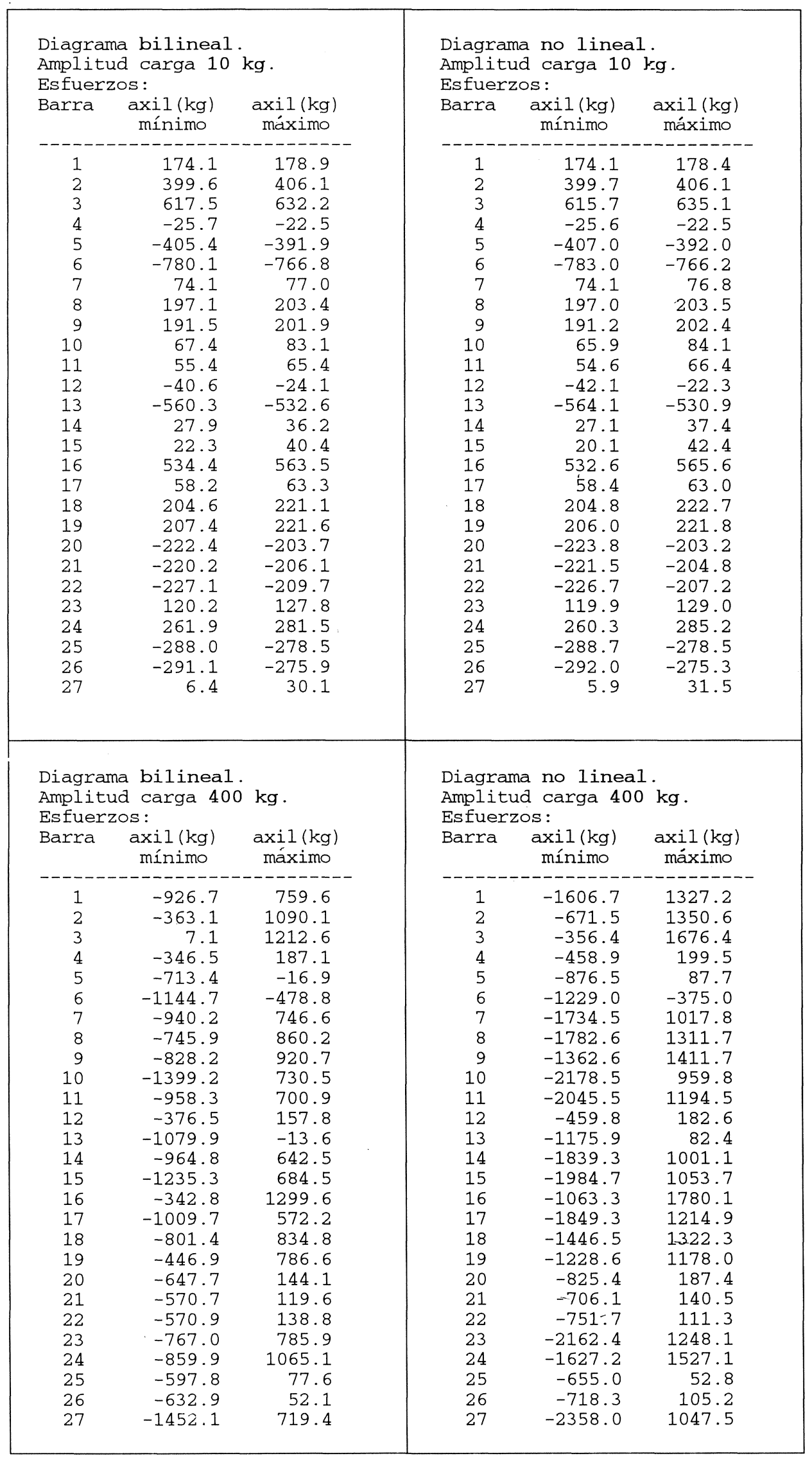

